\title{
Modélisation numérique 3D de la décantation dans les bassins d'assainissement
}

\author{
Gilles ISENMANN ${ }^{1,2}$, Matthieu DUFRESNE ${ }^{1,2}$, José VAZQUEZ $Z^{1,2}$, Robert MOSE ${ }^{1,2}$ \\ ${ }^{l}$ Ecole Nationale du Génie de l'Eau et de l'Environnement de Strasbourg (ENGEES), 1 quai Koch, 67070 Strasbourg, France - e-mail : gilles. \\ isenmann@engees.unistra.fr \\ ${ }_{2}^{2}$ ICube, Mécanique des fluides, Hydraulique urbaine, 2 rue Boussingault, 67000 Strasbourg, France
}

\begin{abstract}
RÉSUMÉ. - L'objectif de cette étude est le développement et la validation d'un outil numérique permettant d'évaluer la performance d'un ouvrage de décantation vis-à-vis de l'abattement des matières en suspension. L'approche Euler-Lagrange est retenue pour la modélisation de l'écoulement et du transport solide. Le modèle numérique développé s'appuie sur la bibliothèque open-source OpenFOAM ${ }^{\circledR}$, enrichie de nouvelles conditions d'interaction particule/paroi afin de restreindre le dépôt aux zones présentant des conditions hydrodynamiques favorables. En particulier, une nouvelle relation est proposée pour le calcul de l'énergie cinétique turbulente seuil en fonction des propriétés de chaque particule. Le modèle numérique est confronté à trois jeux de données expérimentales issues de la littérature et collectées sur des modèles réduits de bassin. L'ensemble de ces expérimentations permettent d'investiguer une large gamme des paramètres représentatifs de l'écoulement (nombres de Froude et de Reynolds) et du transport solide (diamètre adimensionnel). La comparaison des résultats numériques et expérimentaux permet de conclure sur la capacité du modèle à prévoir l'abattement avec une erreur absolue de l'ordre de 5\% lorsque les dépôts ont lieu sur l'ensemble du fond. Dans le cas de dépôts localisés dans des zones préférentielles, la répartition des dépôts est bien reproduite par le modèle et l'abattement est évalué avec une erreur absolue de l'ordre de 10\% (hors cas de particules très peu denses).
\end{abstract}

Mots-clés : Suivi de particules ; transport solide ; dépôt ; matières en suspension ; CFD

\section{Settling efficiency of storm-water tanks using 3D modelling}

\begin{abstract}
The purpose of this study is to develop and validate a numerical tool for evaluating the performance of a settling basin regarding the trapping of suspended matter. The Euler-Lagrange approach was chosen to model the flow

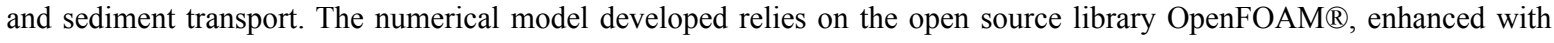
new particle/wall interaction conditions to limit sediment deposition in zones with favourable hydrodynamic conditions. In particular, a new relation is proposed for calculating the turbulent kinetic energy threshold as a function of the properties of each particle. The numerical model is compared to three experimental datasets taken from the literature and collected for scale models of basins. The comparison of the numerical and experimental results permits concluding on the model's capacity to predict the trapping of particles in a settling basin with an absolute error in the region of 5\% when the sediment depositions occur over the entire bed. In the case of sediment depositions localised in preferential zones, their distribution is reproduced well by the model and trapping efficiency is evaluated with an absolute error in the region of $10 \%$ (excluding cases of particles with very low density).
\end{abstract}

Key-words: Particle tracking ;sediment transport ;sediment deposition ; suspended matter ; CFD

\section{INTRODUCTION}

Les eaux de ruissellement transportées dans les réseaux d'assainissement peuvent véhiculer des substances néfastes pour les milieux aquatiques (matières organiques, métaux, hydrocarbures, etc.). Une fraction importante de cette pollution est fixée sur les matières en suspension (MES) [Ashley et al., 2004]. Afin de limiter la pollution rejetée vers le milieu récepteur, différents dispositifs sont mis en place à l'exutoire des réseaux avec l'objectif commun de piéger les MES par décantation. La taille de ces ouvrages peut varier quelques mètres cubes, pour les séparateurs lamellaires ou dynamiques, à la dizaine de milliers de mètres cubes pour les bassins de stockage. Malgré l'utilisation intensive de ces dispositifs, leur fonctionnement est souvent peu connu et leur efficacité vis-à-vis de la décantation est estimée à partir de règles de dimensionnement basées sur l'approche de Hazen [Jarrel-Smith et Friedrich, 2011]. Or, selon la géométrie de l'ouvrage, l'écoulement peut présenter des formes très différentes : recirculations horizontales ou verticales, symétriques ou asymétriques [Kantoush, 2008 ; Dufresne et al., 2009]. Dans ce cas, l'approche de Hazen peut s'avérer incertaine, car en dehors de son domaine d'application, et une autre méthode doit être employée pour évaluer plus précisément l'efficacité de l'ouvrage.

Plusieurs solutions sont disponibles. Une évaluation sur site est envisageable à travers l'instrumentation du dispositif par turbidimétrie ou prélèvements d'échantillons en entrée et en sortie par exemple [Torres, 2008]. Néanmoins cette évaluation n'est possible qu'une fois l'ouvrage construit et son utilisation est inadaptée en phase de dimensionnement. Le fonctionnement d'un ouvrage de décantation peut également 
être étudié en laboratoire [Adamsson et al., 2005]. Toutefois, la mise en place d'un tel dispositif peut s'avérer coûteuse et est réservée à des ouvrages de taille limitée ou des modèles réduits. En revanche, les données expérimentales collectées sur site ou en laboratoire sont indispensables pour valider un modèle numérique $3 \mathrm{D}$, autre solution pour évaluer le fonctionnement d'un ouvrage à moindre coût. De ce fait, cet article s'intéresse au développement et à la validation d'une méthodologie fondée sur la modélisation numérique afin de prédire les performances d'un dispositif de décantation à des fins de dimensionnements.

Le mouvement d'un ensemble de particules solides dans un fluide peut être modélisé par deux méthodes : l'approche Euler-Euler et l'approche Euler-Lagrange. Dans l'approche Euler-Euler, la phase fluide et la phase particulaire sont considérées comme des phases continues et modélisées à partir des équations de Navier-Stokes. Pour l'approche Euler-Lagrange, les équations de Navier-Stokes sont toujours résolues pour la phase fluide et l'équation du mouvement de Newton est utilisée pour déterminer la trajectoire de chaque particule au sein de l'écoulement [Maxey et Riley, 1983]. La concentration en MES dans les eaux de ruissellement étant la plupart du temps inférieure à $1 \mathrm{~g} / 1$ [Bressy, 2010], la phase particulaire n'influence pas l'écoulement et le calcul de l'hydrodynamique peut être découplé du mouvement des particules [Graf et Altinakar, 2000]. L'approche Euler-Lagrange découplée a été employée avec succès dans plusieurs études pour reproduire le taux de d'abattement des particules dans un bassin ainsi que la répartition des dépôts sur le fond [Adamsson et al., 2003 ; Dufresne et al., 2008 ; Yan et al., 2014]. Cette approche est utilisée dans la présente étude.

\section{REVUE BIBLIOGRAPHIQUE}

\section{II.1. L'approche Euler-Lagrange}

La modélisation de l'hydrodynamique dans les bassins est bien maîtrisée. Une approche RANS [Versteeg et Malalasekera, 2007] couplée à un modèle de turbulence de type k-epsilon et ses variantes est suffisante pour reproduire la diversité des formes d'écoulement pouvant exister dans les bassins [Stovin et Saul, 1996 ; Kantoush, 2008 ; Dufresne et al., 2010]. Concernant la modélisation du transport solide, le calcul de la trajectoire d'une particule connaissant les forces agissant sur celle-ci [Maxey et Riley, 1983] est également bien maîtrisé [Adamsson et al., 2003 ; Dufresne et al., 2007 ; Yan et al., 2014]. La principale difficulté liée à ce type de modèle réside dans la condition limite utilisée pour prendre en compte le dépôt ou la remise en suspension d'une particule atteignant la paroi du fond.

\section{II.2. Interaction particule/paroi}

Les conditions d'interaction les plus simples sont les conditions stick et rebound [OpenFOAM, 2016]. Pour une condition de type stick, une vitesse nulle est fixée pour la particule rencontrant la paroi et le calcul de sa trajectoire s'arrête. Pour une condition de type rebound, la particule est remise en suspension dans l'écoulement à partir d'une loi de choc élastique. Ces conditions peuvent pourtant s'avérer inadaptées pour représenter les dépôts sur le fond d'un bassin. En effet, aucun dépôt n'est possible avec une condition rebound, alors que l'utilisation d'une condition stick peut entraîner une surestimation des dépôts puisque toute particule atteignant le fond se dépose. De nouvelles conditions ont alors été proposées afin de restreindre les dépôts aux zones présentant des conditions hydrodynamiques favorables. Toutes ces conditions sont fondées sur la même idée proposant la comparaison d'un paramètre hydrodynamique local avec une valeur seuil.

Adamsson et al. [2003] ont utilisé la contrainte de cisaillement sur le fond comme paramètre hydrodynamique (condition BSS pour Bed Shear Stress). La valeur seuil retenue a été évaluée à environ $0,03 \mathrm{~N} / \mathrm{m}^{2}$ par Stovin et Saul [1994] à partir d'expérimentation sur un modèle réduit de bassin. L'utilisation de cette condition BSS a apporté des améliorations importantes en termes de représentation des zones de dépôt par rapport à une condition stick. Il faut toutefois noter que la valeur seuil utilisée n'est a priori valable que pour le modèle réduit et les particules étudiés expérimentalement par Stovin et Saul [1994] et numériquement par Adamsson et al. [2003].

Une autre approche a été proposée par Dufresne et al. [2009]. Celle-ci est fondée sur un seuillage de l'énergie cinétique turbulente près du fond (condition BTKE pour Bed Turbulent Kinetic Energy). A partir d'expériences sur un modèle réduit en laboratoire, les auteurs ont identifié la gamme $1.10^{-4}-3.10^{-4} \mathrm{~m}^{2} / \mathrm{s}^{2}$ pour la valeur seuil de l'énergie cinétique turbulente. Les dépôts simulés avec la condition BTKE ont montré une bonne cohérence avec les dépôts observés et ont permis une meilleure description par rapport à une condition BSS. Yan et al. [2014] ont appliqué une condition BTKE pour reproduire les dépôts dans un ouvrage de grande taille $\left(32000 \mathrm{~m}^{3}\right)$. Les auteurs ont déterminé une valeur seuil environ dix fois plus faible que celle mis en évidence par Dufresne et al. [2009]. Yan et al. [2014] ont alors proposé la relation $k_{c}=\xi v_{s}{ }^{2}$ afin de déterminer l'énergie cinétique turbulente critique, avec $\xi=$ coefficient d'ajustement ; et $v_{s}=$ vitesse de chute représentative de l'échantillon. Les auteurs ont obtenu les résultats les plus satisfaisants en considérant un coefficient $\xi$ égal à 1 et la vitesse de chute $v_{s, 80}$ (percentile 80 de la distribution des vitesses de chute).

Aussi bien pour les conditions BSS que BTKE, les valeurs seuils proposées ont été ajustées expérimentalement pour un bassin et un échantillon de particules donnés, ce qui les rend difficilement transposables pour d'autres configurations. Dans ce contexte, il serait intéressant de disposer d'une relation permettant le calcul de la valeur seuil associée à chaque particule en fonction de ses propriétés (diamètre et masse volumique).

\section{II.3. Analogie avec les canaux}

De nombreuses études ont été menées sur le transport solide dans les canaux et les rivières. Pour les écoulements faiblement chargés, deux modes de transport peuvent être distingués : le charriage et la suspension. La mise en mouvement par charriage d'une particule au repos sur le fond d'un canal est classiquement fondée sur un seuillage de la contrainte de cisaillement à travers le diagramme de Shields [Buffington, 1999]. Plusieurs auteurs [Brownlie, 1981 ; Van Rijn, 1984] ont proposé des relations analytiques permettant de calculer la contrainte critique de mise en mouvement à partir des propriétés des particules et du fluide. En particulier, Brownlie [1981] a proposé (1) pour déterminer la contrainte critique adimensionnelle $\theta_{c}(2)$ en fonction $\mathrm{du}$ diamètre adimensionnel de la particule $d^{*}(3)$

$$
\begin{gathered}
\theta_{c}=0.22 d^{*-0.9}+0.06 \times 10^{-7.7 d^{*-0.9}}, \\
\theta_{c}=\frac{\tau_{c}}{\left(\rho_{p}-\rho\right) g d_{p}},
\end{gathered}
$$




$$
d^{*}=d_{p}\left[\frac{\rho\left(\rho_{p}-\rho\right) g}{\mu^{2}}\right]^{\frac{1}{3}},
$$

où $\tau_{c}=$ contrainte de cisaillement critique; $\rho_{p}=$ masse volumique de la particule; $\rho=$ masse volumique du fluide; $g=$ accélération de la gravité; et $d_{p}=$ diamètre de la particule .

Lorsque la contrainte de cisaillement critique est dépassée, les particules se déplacent dans un premier temps par charriage à proximité du fond. Le transport par suspension apparait lorsque des éjections turbulentes (burst en anglais) se forment et deviennent suffisamment importantes pour maintenir les particules en suspension dans l'écoulement. Plusieurs critères existent pour caractériser le début de la mise en suspension. Ils permettent d'évaluer la vitesse de frottement seuil $u_{c}^{*}=\left(\tau_{c} / \rho\right)^{1 / 2}$ à partir de laquelle la suspension devient importante. De manière générale, ces critères s'expriment sous la forme générique

$$
\frac{u_{c}^{*}}{w_{s}}=\gamma,
$$

où $w_{s}=$ vitesse de chute de la particule; et $\gamma=$ paramètre constant ou variable selon les auteurs. Le critère de Bagnold correspond à une valeur de $\gamma$ constante égale à 1 [Van Rijn, 1984]. La formulation proposée par Van Rijn [1984] fait intervenir $d^{*}$ et s'exprime par $u_{c}^{*} / w_{s}=4 / d^{*}$ pour $1<d^{*}$ $<10$, et $u_{c}^{*} / w_{s}=0,4$ pour $d^{*}>10$.

Au final, l'idée retenue dans la présente étude est de s'inspirer des formulations obtenues en canaux afin de créer de nouvelles conditions d'interaction entre les particules et les parois du fond qui soient adaptées au cas des bassins. L'objectif est de développer un modèle numérique basé sur l'approche Euler-Lagrange, enrichi de nouvelles conditions d'interaction utilisant les propriétés des particules. Le modèle est ensuite confronté à des données expérimentales issues de la littérature afin d'identifier la condition la plus pertinente pour reproduire l'abattement des particules dans un bassin et la localisation des dépôts sur le fond.

\section{MATÉRIEL ET MÉTHODE}

\section{III.1. Modèle numérique}

\section{III.1.1. Hydrodynamique}

Le mouvement du fluide est décrit à partir des équations de Navier-Stokes [Versteeg et Malalasekera, 2007]. Pour un fluide incompressible, ces équations s'expriment par une équation de conservation de la masse et une équation de conservation du mouvement. La nature multiphasique eau/air de l'écoulement est décrite à partir de la méthode Volume of Fluid [Hirt et Nichols, 1981] ne considérant qu'un seul fluide dont les propriétés (masse volumique et viscosité) varient linéairement avec la fraction volumique $\alpha$. Ce paramètre est un marqueur valant 1 dans la phase eau et 0 dans la phase air. Il est calculé en résolvant une équation de transport [Rusche, 2002].

Les écoulements considérés dans cette étude sont turbulents. Les équations de Navier-Stokes sont ainsi moyennées dans le temps en appliquant une décomposition de Reynolds pour la vitesse et la pression instantanées [Versteeg et Malalasekera, 2007], et un modèle de turbulence est utilisé pour décrire l'effet des vitesses fluctuantes sur l'écoulement moyen. Le modèle $k-\omega$ SST (Shear Stress Transport) avec une loi de paroi standard est retenu. La résolution de ces équations est faite à partir du solveur interFoam [Rusche,
2002], disponible dans la bibliothèque de CFD OpenFOAM ${ }^{\circledR}$ [OpenFOAM, 2016]. Une description complète de ce solveur est fournie dans Deshpande et al. [2012].

\section{III.1.2. Transport solide}

Le mouvement d'une particule dans un fluide est décrit du point de vue lagrangien en résolvant l'équation de Newton (5). Connaissant la somme des forces agissant sur une particule sphérique, il est possible de déterminer l'évolution de la position et de la vitesse de la particule, dit autrement sa trajectoire [Maxey et Riley, 1983].

$$
m_{p} \frac{d \boldsymbol{u}_{\boldsymbol{p}}}{d t}=\boldsymbol{F}_{\boldsymbol{D}}+\boldsymbol{F}_{\boldsymbol{g}}+\boldsymbol{F}_{\boldsymbol{a}},
$$

où $m_{p}=$ masse de la particule; $\boldsymbol{u}_{p}=$ vitesse de la particule; $\boldsymbol{F}_{\boldsymbol{D}}=$ force de traînée; $\boldsymbol{F}_{g}=$ poids déjaugé; et $\boldsymbol{F}_{\boldsymbol{a}}=$ forces additionnelles (gradient de pression, masse ajoutée).

La force de traînée s'exprime

$$
F_{D}=\frac{3}{4} \frac{\rho}{\rho_{p}} \frac{m_{p}}{d_{p}} C_{D}\left(\boldsymbol{u}-\boldsymbol{u}_{\boldsymbol{p}}\right)\left|\boldsymbol{u}-\boldsymbol{u}_{\boldsymbol{p}}\right|,
$$

avec $\rho=$ masse volumique du fluide; $\rho_{p}=$ masse volumique de la particule; $m_{p}=$ masse de la particule; $d_{p}=$ diamètre de la particule; $C_{D}^{p}=$ coefficient de traînée; $\boldsymbol{u}=$ vitesse du fluide; et $\boldsymbol{u}_{\boldsymbol{p}}=$ vitesse de la particule.

Le coefficient de traînée $C_{D}$ est une fonction du nombre de Reynolds particulaire, défini tel que $\mathrm{R}_{\mathrm{p}}=\rho d_{p}\left|\boldsymbol{u}-\boldsymbol{u}_{p}\right| / \mu$ où $\mu=$ viscosité dynamique de l'eau. Pour les faibles $\mathrm{R}_{\mathrm{p}}\left(\mathrm{R}_{\mathrm{p}}<\right.$ $0,1)$, le coefficient $C_{D}$ s'exprime par $C_{D}=24 / R_{p}$. Pour les forts $\mathrm{R}_{\mathrm{p}}\left(\mathrm{R}_{\mathrm{p}}>1000\right)$, le coefficient est constant et vaut 0,44 . Pour les nombres de Reynolds particulaire intermédiaires $\left(0,1<\mathrm{R}_{\mathrm{p}}<1000\right)$, le coefficient $C_{D}$ s'exprime par (7).

$$
C_{D}=\frac{24}{\mathrm{R}_{\mathrm{p}}}\left(1+\frac{1}{6} \mathrm{R}_{\mathrm{p}}^{\frac{2}{3}}\right),
$$

Le poids déjaugé d'une particule $\boldsymbol{F}_{g}$ correspond à la force due à la gravité réduite de la poussée d'Archimède liée au fluide environnant. Il s'exprime sous la forme $\boldsymbol{F}_{g}=m_{p} \boldsymbol{g}$ (1$\left.\rho / \rho_{p}\right)$ où $\boldsymbol{g}=$ accélération de la gravité. Les forces additionnelles $\boldsymbol{F}_{\boldsymbol{a}}$ ne sont pas détaillées ici car elles ne sont pas prises en compte dans la suite du fait de l'impact négligeable sur la trajectoire [Dufresne et al., 2009].

La vitesse du fluide $\boldsymbol{u}$ dans (6) correspond à la vitesse instantanée alors que le résultat d'une simulation hydrodynamique par une méthode RANS fournit une vitesse moyenne $\boldsymbol{U}$. Utiliser la vitesse moyenne pour calculer les trajectoires reviendrait à négliger la nature turbulente de l'écoulement. Or la turbulence a pour effet de détourner les particules de leurs trajectoires voire de les piéger dans des tourbillons pendant une certaine période de temps. Ces phénomènes ne sont donc pas négligeables. Suite à l'utilisation d'un modèle RANS, la nature turbulente de l'écoulement peut être prise en compte en utilisant un modèle de marche aléatoire afin de modéliser la dispersion turbulente des particules. Des tourbillons sont créés aléatoirement et ceux-ci affectent la trajectoire des particules. Dans la pratique, une composante de fluctuation locale $\boldsymbol{u}$ ' est ajoutée à la vitesse moyenne du fluide $\boldsymbol{U}$ à l'endroit où se trouve la particule. La vitesse utilisée dans le calcul des trajectoires est alors $\boldsymbol{u}=\boldsymbol{U}+\boldsymbol{u}$ '. La composante fluctuante est estimée par $|\boldsymbol{u}|=\varphi(2 k / 3)^{0,5}$ où $\varphi=$ nombre aléatoire généré à partir d'une distribution Gaussienne de moyenne 0 et de variance 1 ; et $(2 k / 3)^{0,5}=$ moyenne quadratique des fluctuations de la vitesse $\mathrm{du}$ fluide pour une turbulence isotrope. La direction 
de la vitesse fluctuante est également générée de façon aléatoire. L'algorithme utilisé est disponible dans les sources [OpenFOAM, 2016].

La valeur fluctuante $\boldsymbol{u}$ ' est recalculée à chaque pas de temps de corrélation $t_{\text {turb }}$ et est maintenue constante sinon. Le temps de corrélation est calculé à partir du modèle proposé par Amsden et al. [1989] par $t_{\text {turb }}=\min \left(1 / \omega ; c_{p s} k^{0,5} /(\omega \mid \boldsymbol{U}\right.$ $\left.\left.+\boldsymbol{u}^{\prime}-\boldsymbol{u}_{p} \mid\right)\right)$ où $k$ et $\omega=$ variables turbulentes du modèle $k$ - $\omega$ SST; et $c_{p s}=$ constante empirique égale à 0,16432 . Le premier terme dans la parenthèse correspond à la durée de vie du tourbillon et le deuxième représente le temps nécessaire à la particule pour traverser le tourbillon.

\section{III.2. Nouvelles conditions d'interaction particule/paroi}

Les conditions de type BSS et BTKE sont pertinentes pour modéliser le comportement des particules près du fond d'un bassin. Les valeurs seuils à retenir sont par contre toujours en discussion [Adamsson et al., 2003 ; Dufresne et al., 2009 ; Yan et al., 2014]. Une nouvelle approche, à l'échelle de la particule, est proposée pour le calcul des valeurs seuils $\tau_{c}$ (pour la condition BSS) et $k_{c}$ (pour la condition BTKE). Celles-ci sont calculées pour chaque particule en fonction de ses propriétés physiques telles que le diamètre, la masse volumique et la vitesse de chute.

\section{III.2.1. Condition BSS}

La condition BSS se base sur la comparaison de la contrainte de cisaillement au niveau de la paroi avec une contrainte seuil $\tau_{c}$. La valeur seuil est calculée pour chaque particule en fonction de son diamètre adimensionnel $d^{*}(3)$ en s'appuyant sur l'interpolation du diagramme de Shields proposée par Brownlie [1981] dans (1). Cette condition est nommée «BSS Brownlie » pour la suite de l'étude.

\section{III.2.2. Condition BTKE}

\section{Obtention d'une relation}

La relation permettant le calcul de l'énergie cinétique turbulente seuil $k_{c}$ est déduite des critères utilisés pour caractériser le début de la mise en suspension en canaux (4). La vitesse de frottement est par définition

$$
u_{c}^{*}=\sqrt{\frac{\tau_{c}}{\rho}}
$$

De plus, la contrainte de cisaillement peut s'exprimer comme une fonction linéaire de l'énergie cinétique turbulente [Pope et al., 2006] par la relation

$$
\tau_{c}=\rho C k_{c},
$$

où $C=$ constante proche de 0,20 .

En combinant (8) et (9) et en injectant (4), on obtient une relation entre l'énergie cinétique turbulente seuil et la vitesse de chute de la particule : $k_{c}=\gamma^{2} \cdot w_{s}^{2} / C$. En posant $\xi=$ $\gamma^{2} / C$, il vient

$$
k_{c}=\xi w_{s}^{2},
$$

Contrairement à la formulation de Yan et al. [2014], le paramètre $\xi$ n'est pas un coefficient d'ajustement. Il est déduit des critères caractérisant la mise en suspension des particules dans un canal. En s'appuyant sur le critère de Bagnold $(\gamma=$ 1), il vient une valeur de $\xi$ constante et égale à 5 . Le critère de Van Rijn [1984] implique quant à lui une valeur de $\xi$ variable en fonction du diamètre adimensionnel $d^{*}$ telle que

$$
\xi=\frac{80}{d^{* 2}},
$$

pour $1<d^{*}<10$, et $\xi=0,8$ pour $d^{*}>10$.

\section{Prolongement du critère de Van Rijn[1984]}

En s'appuyant sur le critère de Van Rijn [1984], le paramètre $\xi$ n'est pas défini pour les $d^{*}<1$. Deux possibilités vont être investiguées pour le prolongement. La première consiste à conserver (11) pour les $d^{*}<1$. Cette condition sera nommée «BTKE Van Rijn » dans la suite de l'étude. La deuxième repose sur l'estimation de la valeur de $\xi$ à partir du diagramme de Shields (1) et de (9) et (10). La vitesse de chute $w_{s}$ est calculée à partir de la formule de Stokes [Jarrel-Smith et Friedrich, 2011], celle-ci étant valide pour les particules considérées ici $\left(d^{*}<2\right)$. Le choix de l'utilisation du diagramme de Shields pour les $d^{*}<2$ est justifié car les critères caractérisant le début du charriage et le début de la suspension sont confondus pour cette gamme de $d^{*}$ [Van Rijn, 1984]. La courbe $\xi=320 / d^{* 4}$ fournit une approximation acceptable pour les $d^{*}$ compris entre 0,1 et 2,5 . Afin d'assurer la continuité dans le calcul de $\xi$, les relations suivantes sont proposées $\xi=320 / d^{* 4}$ pour $0,1<d^{*}<2$, $\xi=80 / d^{* 2}$ pour $2<d^{*}<10$, et $\xi=0,8$ pour $d^{*}<10$. Cette condition sera nommée «BTKE Van Rijn prolongé » dans la suite de l'étude.

\section{Calcul de la vitesse de chute}

La vitesse de chute d'une particule $w_{s}$ utilisée dans (10) est calculée en résolvant par itérations le système formé par les équations suivantes

$\frac{w_{s}}{\sqrt{\left(\frac{\rho_{p}}{\rho}-1\right) g d_{p}}}=\sqrt{\frac{4}{3 C_{D}}} ; R e_{p}=\frac{\rho d_{p} w_{s}}{\mu} ; C_{D}=A_{1}+\frac{A_{2}}{R e_{p}}+\frac{A_{3}}{R e_{p}^{2}}$

où $\mathrm{A} 1, \mathrm{~A} 2, \mathrm{~A} 3$ = constantes dont les valeurs dépendent de $\operatorname{Re}_{\mathrm{p}}$ [Morsi et Alexander, 1972].

\section{III.3. Expérimentations}

La méthodologie est appliquée à trois séries d'expériences réalisées sur des modèles réduits de bassins par Frey et al. [1993], Stovin et Saul [1994] et Dufresne et al. [2009]. La confrontation des résultats numériques avec les mesures expérimentales permet de vérifier la capacité du modèle à reproduire les phénomènes en jeu dans le transport solide en bassin à travers deux informations : le taux d'abattement des particules dans l'ouvrage et les zones préférentielles de dépôt sur le fond. Les trois séries d'expériences présentent des caractéristiques permettant d'appliquer le modèle sur une large gamme de particules et de configurations hydrauliques. Les paramètres des expériences sont résumés dans le Tableau 1.

Les expériences de Frey et al. [1993] conduisent à la validation du modèle pour des conditions hydrauliques où des dépôts peuvent avoir lieu sur l'ensemble du fond de l'ouvrage. L'objectif principal est donc la vérification de la capacité du modèle à reproduire le taux d'abattement des particules dans l'ouvrage. Dans les expériences de Stovin et Saul [1994], les conditions hydrodynamiques sont telles que des zones vierges de tout dépôt apparaissent sur le fond du bassin. L'échantillon de particules utilisé couvre des $d^{*}$ entre 0,15 et 2,5, ces expériences sont donc bien adaptées pour étudier, en plus du taux d'abattement, les nouvelles conditions d'interaction particule/paroi, en particulier le prolongement du critère de Van Rijn [1984] pour les petits $d^{*}$ 
Tableau 1 : Paramètres des jeux de données expérimentales de la littérature

\begin{tabular}{|c|c|c|c|}
\hline Expériences & Frey et al. $\mathbf{~ ( 1 9 9 3 )}$ & Stovin et Saul (1994) & Dufresne et al. (2009) \\
\hline Nombres de Froude & $0,01-0,05$ & $0,01-0,06$ & $0,002-0,03$ \\
\hline Nombres de Reynolds & $4400-4800$ & $4400-16500$ & $1300-7000$ \\
\hline $\boldsymbol{\rho}_{\boldsymbol{p}} / \boldsymbol{\rho}$ & 2,650 & 1,453 & 1,034 \\
\hline $\mathbf{d}^{*}{ }_{50}$ & 1,$3 ; 1,5 ; 1,8 ; 2,1 ; 2,5$ & 0,77 & 5,12 \\
\hline Dépôts & Sur 1'ensemble du fond & Dans des zones localisées & Dans des zones localisées \\
\hline
\end{tabular}

$\left(d^{*}<2\right)$. Dans les expériences de Dufresne et al. [2009], les particules utilisées possèdent des $d^{*}$ entre 2,5 et 10 . Comme pour les expériences de Stovin et Saul [1994], les conditions hydrodynamiques dans le bassin sont telles que le dépôt est impossible dans certaines zones. Ces expériences permettent donc d'étudier la pertinence des conditions d'interaction particule/paroi pour des $d^{*}$ plus importants $\left(\mathrm{d}^{*}>2\right)$.

\section{III.3.1. Les expériences de Frey et al. [1993]}

\section{Protocole et résultats expérimentaux}

Les expériences menées par Frey et al. [1993] s'intéressent au transport de particules de sable dans quatre modèles réduits de bassin de décantation. Les configurations géométriques et hydrauliques étudiées sont basées sur une similitude de Froude par rapport à des décanteurs de vignoble (échelle 1/8). Les détails quant à la forme et aux dimensions de l'ouvrage sont disponibles dans Frey et al. [1993]. Deux débits $(1,1$ et 2,2 1/s) sont testés en entrée de l'ouvrage. Pour chacune des combinaisons géométriques et hydrauliques, cinq expériences de transport solide sont réalisées, chacune correspondant à un échantillon de sable fin. Les échantillons de sable sont caractérisés par le diamètre médian de la distribution granulométrique $\left(d_{50}=51 ; 60\right.$; $70 ; 84 ; 101 \mu \mathrm{m})$. Au final, le pourcentage massique en sortie (égal à la masse sortie divisée par la masse injectée) est évalué pour chaque expérience. L'efficacité (égale à la masse décantée divisée par la masse injectée) est présentée sur la Figure 1 pour chacune des 34 expériences. La précision sur le pourcentage massique en sortie est évaluée à environ 5\% [Frey et al., 1993].

\section{Modélisation numérique de l'écoulement et du transport solide}

Les sept combinaisons géométriques et hydrauliques sont simulées numériquement avec le solveur interFoam présenté précédemment. Les quatre géométries sont maillées avec l'outil snappyHexMesh [OpenFOAM, 2016]. Des mailles de type « cut-cell » d'environ 7,5 $\mathrm{mm}$ de côtés et $2,5 \mathrm{~mm}$ de hauteur sont choisies (soit un $\mathrm{y}^{+}$de l'ordre de 1 à 3 ), conduisant à un domaine de calcul composé de 500000 à 600000 cellules selon la géométrie. La vitesse est imposée sur la face d'entrée de l'ouvrage afin de reproduire les débits injectés expérimentalement. La condition limite en sortie et sur la partie supérieure du domaine de calcul est une pression atmosphérique. Le modèle de turbulence $k-\omega$ SST est utilisé avec des lois de parois standards [Versteeg et Malalasekera, 2007 ; OpenFOAM, 2016]. La convergence du calcul est vérifiée en s'appuyant sur la stabilité au cours du temps du bilan de masse en sortie et du champ de vitesses dans différents plans longitudinaux et transversaux.

Le modèle de suivi de particule présenté précédemment est appliqué aux sept combinaisons géométriques et hydrauliques. Pour chaque échantillon de sable, une distribution de
Rosin - Rammler est interpolée afin de reproduire la répartition granulométrique expérimentale. Après avoir vérifié que ce nombre était suffisant pour obtenir des résultats statistiquement représentatifs, dix mille particules de masse volumique $2650 \mathrm{~kg} / \mathrm{m}^{3}$ sont injectées dans le flux hydraulique. Afin de reproduire le mode d'injection utilisé expérimentalement par Frey et al. [1993], la surface d'injection est définie par un disque de $8 \mathrm{~mm}$ de diamètre positionné à l'amont immédiat de la rupture de pente. Le centre du disque est centré par rapport aux parois latérales et situé à $10 \mathrm{~mm}$ du fond du canal d'alimentation. Les forces prises en compte pour le calcul des trajectoires des particules sont la force de traînée et le poids déjaugé. La nature turbulente de l'écoulement est modélisée à partir du modèle de dispersion stochastique présenté précédemment. Une condition rebound est utilisée pour l'entrée et les parois latérales. Une condition escape [OpenFOAM, 2016] est appliquée à la sortie et l'atmosphère. Concernant le fond de l'ouvrage, l'utilisation de la condition stick est a priori suffisante puisque d'une part, les dépôts observés expérimentalement ne sont pas localisés dans certaines régions du fond [Frey et al., 1993], et d'autre part, les auteurs ont montré qu'en recouvrant le fond du décanteur de particules et en amenant progressivement le débit à sa valeur nominale, il n'y avait pas de remise en suspension (aucune particule collectée en sortie de l'ouvrage). Les nouvelles conditions d'interaction particule/paroi sont toutefois utilisées pour déterminer si une d'entre elles permet d'obtenir des résultats plus cohérents qu'avec la condition stick ou à défaut vérifier qu'elles fournissent des résultats similaires. Les conditions aux limites utilisées sont donc : stick, « BSS Brownlie », « BTKE Van Rijn », « BTKE Van Rijn prolongé » et une condition BTKE avec un coefficient $\xi$ fixe. Pour cette dernière condition, les valeurs $\xi=1$ [Yan et al., 2014] et $\xi=5$ (critère de Bagnold) sont étudiées.

\section{III.3.2. Les expériences de Stovin et Saul [1994]}

\section{Protocole et résultats expérimentaux}

Les expériences de Stovin et Saul [1994] ont été menées sur un modèle réduit de bassin rectangulaire en similitude de Froude (échelle 1/15) avec un ouvrage de stockage typiquement rencontré sur les réseaux d'assainissement unitaires. La forme et les dimensions de l'ouvrage sont détaillées dans Stovin [1996]. Une plage de débits entre 4 et 16 1/s est investiguée pour deux hauteurs d'eau fixes dans le bas$\sin (0,2$ et $0,3 \mathrm{~m})$. Les particules injectées sont des noyaux d'olives broyés de masse volumique $1453 \mathrm{~kg} / \mathrm{m}^{3}$. La distribution granulométrique s'étend de 5 à $150 \mu \mathrm{m}$ avec un diamètre médian à $47 \mu \mathrm{m}$. L'efficacité de l'ouvrage vis-à-vis de l'abattement des particules est déterminé à partir de la concentration en sortie et de la concentration en entrée, ces deux valeurs étant estimées à partir de mesures de turbidité. Trois essais sont effectués pour chaque configuration 
hydraulique. Les résultats expérimentaux présentent donc trois valeurs d'efficacité pour un débit donné. Les mesures expérimentales sont résumées sur la Figure 2. Par ailleurs, la localisation des dépôts sur le fond du bassin est relevée par des observations à l'œil nu et des photographies puis reportée sur un croquis pour trois expériences (Figure 3).

\section{Modélisation numérique de l'écoulement et du transport solide}

La méthodologie suivie pour la modélisation de l'écoulement et du transport solide est analogue à celle présentée pour la modélisation des expériences de Frey et al. [1993]. Tous les détails sont disponibles dans Isenmann [2016]. Pour le fond de l'ouvrage, les conditions d'interaction particule/paroi investiguées sont : stick, " BSS Brownlie », «BTKE Van Rijn », « BTKE Van Rijn prolongé ». Les conditions BTKE utilisant une valeur de $\xi$ fixe ne sont pas utilisées, une étude préliminaire de la distribution d'énergie cinétique turbulente sur le fond ayant montré qu'aucune particule ne pourrait se déposer étant données les valeurs seuils calculées [Isenmann, 2016].

\section{III.3.3. Les expériences de Dufresne et al. (2009)}

\section{Protocole et résultats expérimentaux}

Les expériences menées par Dufresne et al. [2009] ont été réalisées sur un modèle réduit de bassin rectangulaire. Deux configurations ont été étudiées : une sortie par une conduite en partie basse du bassin (géométrie A) et une sortie par surverse (géométrie B). La forme et les dimensions de l'ouvrage sont détaillées dans Dufresne [2008]. Une plage de débits entre 1 et $5 \mathrm{l} / \mathrm{s}$ est investiguée pour différentes hauteurs d'eau entre 8 et $40 \mathrm{~cm}$. Les particules injectées sont des billes de polystyrol de couleur blanche dont la distribution granulométrique s'étend entre 350 et $1400 \mu \mathrm{m}$ avec un diamètre médian à $738 \mu \mathrm{m}$. La masse volumique est évaluée à $1034 \mathrm{~kg} / \mathrm{m}^{3}\left(+/-19 \mathrm{~kg} / \mathrm{m}^{3}\right)$ par pycnométrie. En fin d'expérience, les particules décantées et récupérées en sortie sont réintroduites dans une colonne de mesure afin d'évaluer l'efficacité de l'ouvrage. De plus, des prises de vues (photographies et films) du fond du bassin sont réalisées pour chaque expérience afin de suivre l'évolution des dépôts.

\section{Modélisation numérique de l'écoulement et du transport solide}

La méthodologie suivie pour la modélisation de l'écoulement et du transport solide est analogue à celle présentée pour la modélisation des expériences de Frey et al. [1993] et Stovin et Saul [1994]. Tous les détails sont disponibles dans Isenmann [2016]. Le choix est fait de ne s'intéresser ici qu'aux résultats sur les zones de dépôts afin d'étudier la pertinence des conditions d'interaction entre les particules et le fond pour la gamme haute des diamètres adimensionnels $\left(d^{*}>2\right)$. Les conditions étudiées sont : stick, «BSS Brownlie », «BTKE Van Rijn » et BTKE avec une valeur de $\xi$ fixe (égal à 1 et 5 ). Il faut noter que la condition « BTKE Van Rijn prolongé » est équivalente à la condition « BTKE Van Rijn » pour la gamme des $d^{*}$ associés à ces expériences $\left(d^{*}{ }_{\min }=2,5\right)$.
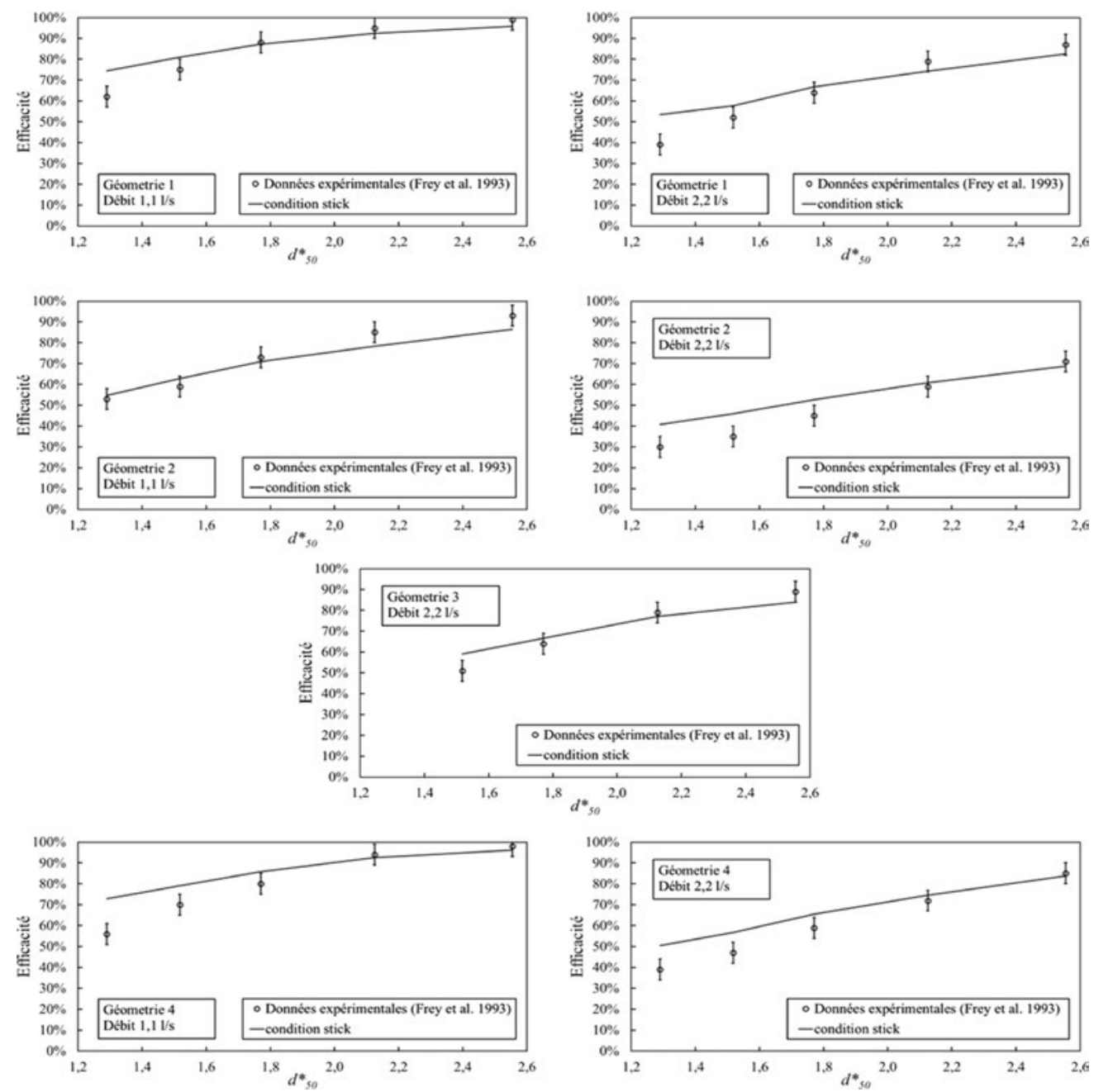

Figure 1 : Comparaison des résultats numériques avec les données expérimentales de Frey et al. [1993] 


\section{RÉSULTATS ET DISCUSSION}

\section{IV.1. Comparaison des résultats numériques et expérimentaux}

\section{IV.1.1. Les expériences de Frey et al. [1993]}

Lorsque l'ensemble des particules sont déposées ou ont quitté l'ouvrage par la sortie, le pourcentage massique en sortie peut être calculé. La Figure 1 montre ainsi la comparaison des résultats numériques et expérimentaux pour les 34 simulations effectuées avec la condition stick. Chaque graphique correspond à une configuration géométrique et hydraulique pour les différents échantillons de sable étudiés. Les échantillons sont représentés par leur diamètre adimensionnel médian $d^{*}{ }_{50}$. L'écart absolu entre la valeur calculée et celle mesurée expérimentalement est en moyenne de $+/-6 \%$ sur l'efficacité. L'écart est plus important pour les faibles valeurs de $d^{*}$, celui-ci pouvant atteindre $17 \%$ au maximum. De manière générale, le modèle numérique reproduit fidèlement le taux d'abattement des particules dans l'ouvrage, la plupart des valeurs étant à l'intérieur des barres d'incertitude expérimentales. Les résultats numériques obtenus pour les autres conditions d'interaction particule/paroi ne sont pas détaillés ici et sont disponibles dans Isenmann [2016]. On peut toutefois noter que les conditions «BSS Brownlie », «BTKE Van Rijn » et «BTKE Van Rijn prolongé » fournissent des résultats similaires à la condition stick et sont donc bien adaptées pour ce cas où des dépôts recouvrent l'ensemble du fond. Par contre les deux conditions BTKE utilisant une valeur de $\xi$ fixe (égale à 1 ou 5) apparaissent comme étant trop contraignante vis-à-vis du dépôt et entrainent ainsi des efficacités sous-estimées la plupart du temps.

\section{IV.1.2. Les expériences de Stovin et Saul [1994]}

En fin de simulation, l'efficacité du bassin est calculée pour chacune des conditions aux limites investiguées pour le fond de l'ouvrage. La Figure 2 illustre la comparaison des efficacités numériques et expérimentales en fonction du débit d'alimentation pour les hauteurs d'eau $0,20 \mathrm{~m}$ et $0,30 \mathrm{~m}$. Les mesures expérimentales pouvant présenter une large gamme d'efficacité pour un même débit (par exemple entre $8 \%$ et $27 \%$ à $11,5 \mathrm{l} / \mathrm{s}$ pour la hauteur d'eau $0,20 \mathrm{~m}$ sur la Figure 2), les écarts sont calculés par rapport à la valeur moyenne des trois mesures expérimentales.

Les résultats sur l'efficacité montrent une forte sensibilité à la condition d'interaction utilisée. La condition stick entraine une surestimation de l'efficacité de l'ordre de 20-35\% dans la plupart des cas, sauf pour les faibles débits. L'efficacité est également surestimée avec la condition «BSS Brownlie », avec tout de même un écart moins important avec les mesures expérimentales (environ 10-20\%). A l'inverse, la condition "BTKE Van Rijn » entraine une sous-estimation de l'efficacité pour l'ensemble des expériences. L'écart avec les mesures peut atteindre 30\% (débit faible et hauteur d'eau $0,30 \mathrm{~m}$ ). Enfin, la condition «BTKE Van Rijn prolongé » fournit des efficacités tantôt supérieures et tantôt inférieures aux mesures expérimentales, l'écart étant toujours inférieur à $10 \%$, sauf pour le débit le plus faible $(15 \%)$. Les résultats obtenus avec cette dernière condition sont cohérents avec la gamme des mesures expérimentales pour la plupart des expériences.

L'autre point essentiel pour déterminer la condition d'interaction la plus efficace concerne la localisation des dépôts sur le fond de l'ouvrage. Les dépôts en fin de simulation sont présentés sur la Figure 3 pour chaque condition d'interaction et sont comparés avec les dépôts observés par Stovin [1996]. Les comparaisons sont faites pour les trois expériences pour lesquelles les dépôts observés expérimentalement sont disponibles, à savoir celles réalisées pour la hauteur d'eau $0,20 \mathrm{~m}$ et les débits 4,$28 ; 10,23$ et $16,03 \mathrm{l} / \mathrm{s}$.

Les dépôts obtenus avec les conditions stick et «BTKE Van Rijn » confirment la faiblesse de ces conditions, déjà mise en évidence lors de la comparaison des efficacités. En effet, la condition stick entraîne des dépôts sur la totalité $\mathrm{du}$ fond et ne reproduit donc pas les zones de dépôts observées expérimentalement. La condition «BTKE Van Rijn» sous-estime quant à elle fortement le dépôt, voire le limite à seulement quelques particules pour les débits importants. Les dépôts obtenus avec la condition «BSS Brownlie » reproduisent particulièrement bien les zones de dépôts observées pour le débit le plus faible. Par contre, pour les deux autres débits, un nombre important de particules se déposent dans des zones qui devraient être vierges de tout dépôt. Enfin, les dépôts obtenus avec la condition «BTKE Van Rijn prolongé » sont en bon accord avec les observations de Stovin [1996]. Les parties vierges de dépôt pour le débit faible sont relativement bien reproduites, malgré une légère surestimation de la taille de la bande longitudinale exempte de dépôt le long du jet principal. Pour le débit intermédiaire, les dépôts dans les coins amont gauche et droit sont bien mis en évidence. Le dépôt isolé au centre du bassin est par contre moyennement reproduit. Pour le débit le plus important, le modèle reproduit les dépôts dans les coins amont gauche et droit, le reste du fond étant vierge de dépôt.

\section{IV.1.3. Les expériences de Dufresne et al. [2009]}

Les dépôts observés sur le fond du bassin ont été photographiés pour chaque expérience par Dufresne [2008]. Les résultats présentés par la suite illustre une partie des comparaisons entre dépôts numériques et expérimentaux. La totalité des expériences est disponible dans Isenmann [2016]. La comparaison est faite pour les trois conditions d'interaction particule/paroi présentant un intérêt, à savoir la condition BTKE avec une valeur de $\xi$ fixe égale à 1 (nommée « BTKE $\xi=1$ par la suite), la condition «BTKE Van Rijn » et la condition «BSS Brownlie ». Les résultats obtenus avec les
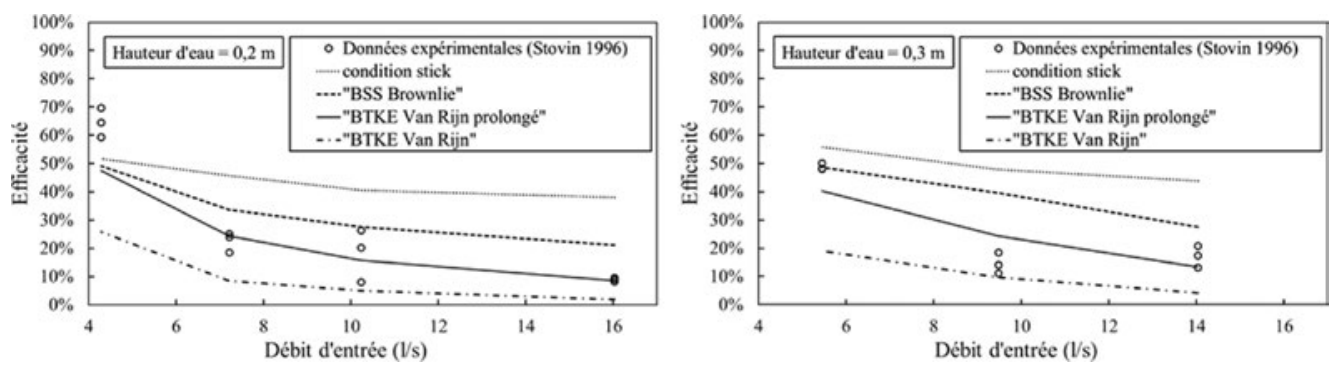

Figure 2 : Comparaison des efficacités numériques avec les données expérimentales de Stovin [1996] 


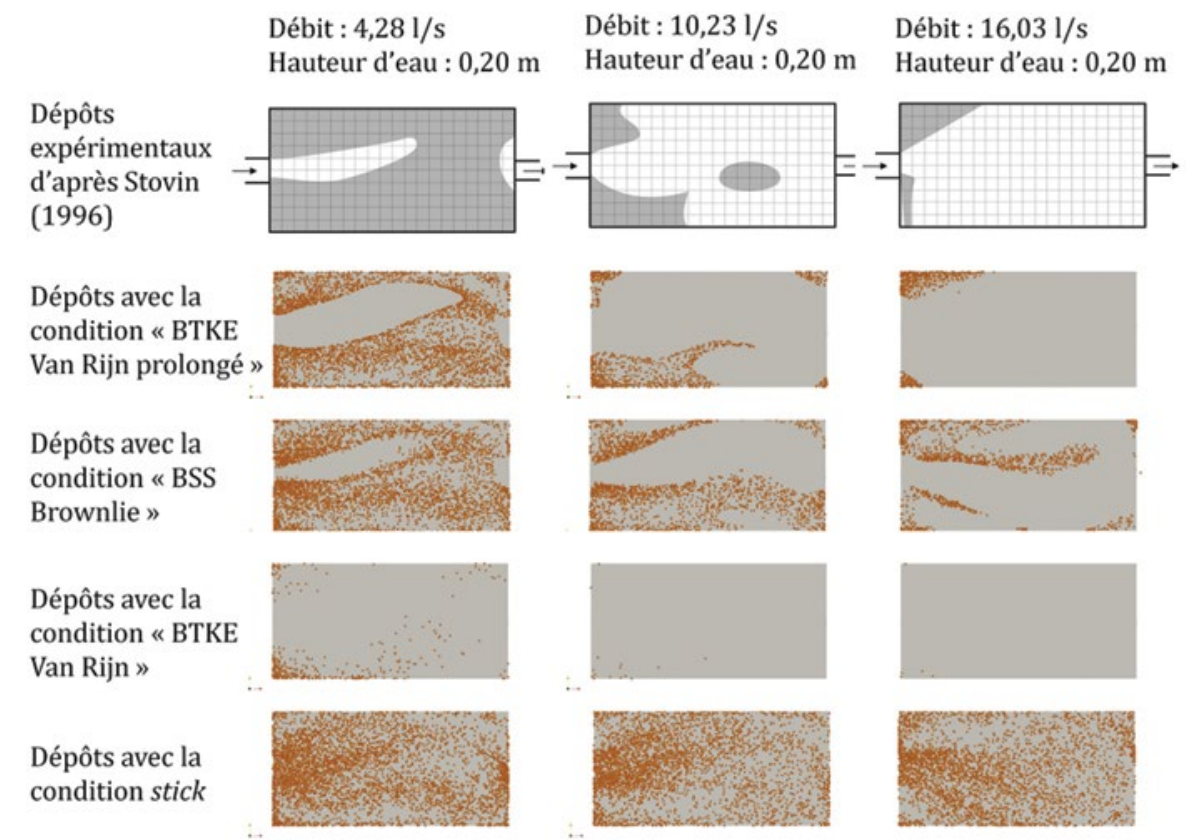

Figure 3 : Comparaison des dépôts numériques avec les données expérimentales de Stovin [1996]

conditions stick et BTKE avec une valeur de $\xi$ fixe égale à 5 ne sont pas détaillés car celles-ci entraînent une importante surestimation des zones de dépôts pour l'ensemble des expériences [Isenmann, 2016].

La Figure 4 illustre les zones préférentielles de dépôts pour une expérience sur la géométrie A (débit $3 \mathrm{l} / \mathrm{s}$, hauteur d'eau $35 \mathrm{~cm}$ ). Deux zones de dépôts quasi-symétriques sont observées dans les coins amont du bassin. Celles-ci se prolongent vers l'aval, le long des parois latérales, en diminuant progressivement de largeur jusqu'à s'estomper. Les zones préférentielles de dépôts sont particulièrement bien reproduites dans les coins amont et le long des parois latérales pour la condition « BTKE Van Rijn ». Deux zones de dépôt restreintes sont cependant mises en évidence à proximité des coins aval du bassin alors que cette zone est vierge de dépôt par l'observation. Les conditions «BTKE $\xi=1$ » et «BSS Brownlie » reproduisent les dépôts de part et d'autre de la conduite d'entrée mais sous-estiment globalement la taille de la zone de dépôt dans les coins amont et le long des parois. On remarque par ailleurs la présence de deux bandes de dépôt au centre des deux recirculations symétriques de l'amont vers l'aval du bassin pour la condition "BSS Brownlie », cette zone ne présentant pourtant aucun dépôt expérimentalement.

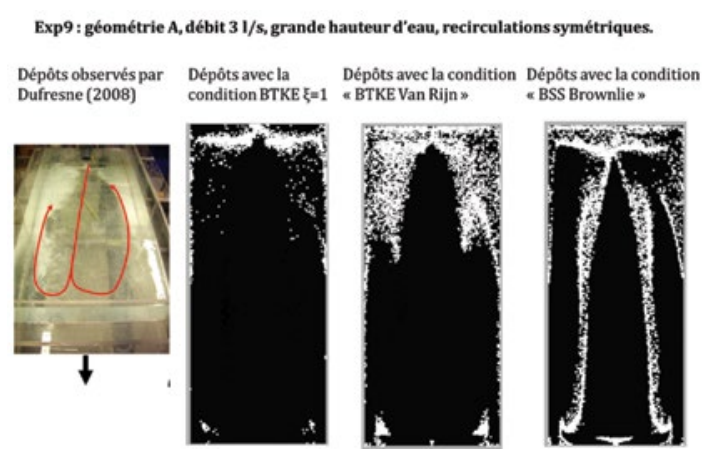

Figure 4 : Comparaison des dépôts numériques avec les observations de Dufresne [2008] pour la géométrie A
La Figure 5 illustre les dépôts observés par Dufresne [2008] pour une expérience sur la géométrie $\mathrm{B}$ avec un débit relativement faible (1 $1 / \mathrm{s})$. Une zone vierge de dépôt est mise en évidence au niveau du jet principal. Les particules se déposent autour du jet puis dans la partie à l'aval immédiat de la zone vierge de dépôt. Les conditions «BTKE Van Rijn » et «BSS Brownlie » permettent une bonne reproduction des dépôts de part et d'autre du jet principal ainsi qu'à l'aval de la zone vierge de dépôt. La taille de celle-ci est par ailleurs légèrement sous-estimée avec la condition «BTKE Van Rijn » et surestimée avec la condition «BSS Brownlie ». La condition «BTKE $\xi=1$ » met en évidence la zone exempte de dépôt mais la répartition des particules de part et d'autre du jet est mal représentée. On remarque également une trop forte accumulation des dépôts au niveau de la paroi à l'aval du bassin par rapport aux observations expérimentales.

De manière générale, la condition «BTKE Van Rijn » (équivalente à « BTKE Van Rijn prolongé » pour la gamme de $d^{*}$ ) permet une bonne représentation des zones préférentielles de dépôts pour l'ensemble des expériences. Les zones vierges de particules sont bien mises en évidence, en particulier pour les expériences avec des grandes hauteurs d'eau $(>30 \mathrm{~cm})$. Les zones vierges de dépôt sont dans l'ensemble

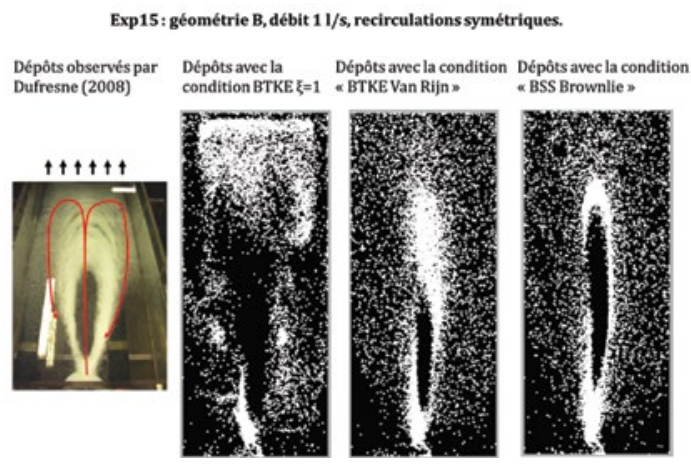

Figure 5 : Comparaison des dépôts numériques avec les observations de Dufresne [2008] pour la géométrie B 
mal représentées par la condition « BSS Brownlie ». En effet, celle-ci entraine des bandes de dépôts aux centres des recirculations symétriques qui ne sont pas observées expérimentalement. La condition « BTKE $\xi=1$ » entraîne la plupart du temps une reproduction imprécise des zones de dépôts. Cette condition apparait comme trop contraignante, dans le sens où elle sous-estime les zones de dépôts des particules. Les parties du fond vierges de dépôt sont mises en évidence mais sont souvent trop étendues.

\section{IV.2. Discussion sur l'efficacité et les zones de dépôts}

Le modèle développé a été appliqué à trois séries d'expériences menées sur des modèles réduits de bassins dans le but de vérifier la capacité du modèle à reproduire les phénomènes en jeu dans le transport solide en ouvrage de décantation. Les deux informations essentielles à la validation du modèle sont : le taux d'abattement des particules dans l'ouvrage et les zones préférentielles de dépôt sur le fond.

La capacité du modèle à reproduire l'efficacité d'un ouvrage a tout d'abord été vérifiée par confrontation avec les données expérimentales de Frey et al. [1993]. Pour les 34 expériences, couvrant des $d^{*}$ entre 0,5 et 5 , l'écart moyen entre les efficacités calculées et mesurées est d'environ $6 \%$, la plupart des valeurs étant à l'intérieur des barres d'incertitude expérimentales. Etant données les conditions hydrodynamiques et les propriétés des particules, les dépôts pouvaient se faire sur l'ensemble du fond du bassin. L'impact des nouvelles conditions d'interaction vis-à-vis des zones préférentielles de dépôt n'a donc pas été étudié pour ces expériences puisqu'elles fournissent, pour la plupart, des résultats identiques à ceux obtenus avec la condition stick (les deux conditions BTKE utilisant une valeur de $\xi$ fixe ont conduit à des résultats incohérents avec les observations expérimentales et ont ainsi montré leurs limites).

La condition stick est par contre inadaptée dans le cas où des zones vierges de dépôt existent sur le fond de l'ouvrage comme pour les expériences de Stovin et Saul [1994] et Dufresne et al. [2009]. Les nouvelles conditions d'interaction implémentées ont donc été étudiées en s'appuyant sur ces deux jeux de données expérimentales. Dans un premier temps, la gamme de $d^{*}$ entre 0,1 et $2,5\left(d^{*}{ }_{50}=0,77\right)$ a été investiguée à partir des expériences de Stovin et Saul [1994]. La condition « BTKE Van Rijn prolongé » a permis d'obtenir des résultats satisfaisants, aussi bien pour les efficacités que pour la représentation des zones préférentielles de dépôt sur le fond. Les autres conditions BTKE, à savoir «BTKE Van Rijn » et les deux conditions BTKE utilisant une valeur de $\xi$ fixe (1 ou 5), ont toutes montré leur incapacité à reproduire les mesures et les observations expérimentales pour la gamme de $d^{*}$ investiguée. La condition fondée sur un seuillage de la contrainte de cisaillement (« BSS Brownlie ») a permis d'obtenir des efficacités acceptables mais a montré sa limite dans la reproduction des zones de dépôt pour les débits importants. Dans un second temps, la gamme de $d^{*}$ entre 2,5 et $10\left(d^{*}{ }_{50} \approx 5\right)$ a été étudiée en s'appuyant sur les données expérimentales de Dufresne et al. [2009]. Là aussi, la condition " BTKE Van Rijn prolongé » (équivalente « BTKE Van Rijn » pour cette gamme de $d^{*}$ ) a fourni les résultats les plus satisfaisants. Les deux conditions BTKE utilisant une valeur de $\xi$ fixe (1 ou 5) se sont montrées insuffisantes pour reproduire fidèlement les zones de dépôt sur le fond, celles-ci sont soit sous-estimées $(\xi=1)$ soit surestimées $(\xi=5)$. Les zones de dépôts obtenues avec la condition « BSS Brownlie » sont mal reproduites, en particulier pour les hauteurs d'eau importantes dans le bassin.
Au final, la condition d'interaction « BTKE Van Rijn prolongé » s'avère être la plus pertinente pour des $d^{*}$ entre 0,1 et 10 . De manière générale, celle-ci a permis de reproduire de manière satisfaisante le taux d'abattement des particules et les zones préférentielles de dépôt pour les trois séries d'expériences sur des modèles réduits de bassin.

\section{CONCLUSIONS}

L'objectif de cette étude était la mise au point d'un outil d'évaluation des performances d'un ouvrage de décantation vis-à-vis de l'abattement des matières en suspension (efficacité de l'ouvrage). Une méthode numérique utilisant l'approche Euler-Lagrange a été retenue pour la modélisation de l'écoulement et du transport solide. Un solveur pour la modélisation lagrangienne a ainsi été créé en s'appuyant sur la bibliothèque OpenFOAM ${ }^{\circledR}$. Celui-ci est développé pour des écoulements à surface libre puisqu'il s'appuie sur les résultats hydrodynamiques d'un solveur multiphasique utilisant une méthode Volume of Fluid.

De nouvelles conditions d'interaction entre les particules et les parois, de type BSS et BTKE, ont été proposées afin de déterminer les valeurs seuils à l'échelle de la particule. En particulier, une nouvelle relation a été présentée pour le calcul de l'énergie cinétique turbulente seuil en fonction des propriétés de la particule (diamètre et masse volumique). Cette relation repose sur l'évaluation de $k_{c}=\xi \cdot w_{s}{ }^{2}$ avec $\xi$ calculé sur la base des critères (prolongés) de Van Rijn [1984] en fonction du $d^{*}$, et $w_{s}$ calculée par un bilan des forces agissant sur la particule.

Le modèle numérique a été appliqué à trois séries d'expériences menées sur des modèles réduits de bassins [Frey et al., 1993 ; Stovin et Saul, 1994 ; Dufresne et al., 2009] afin de vérifier la capacité du modèle à reproduire les phénomènes en jeu dans le transport solide en ouvrage de décantation. Les résultats obtenus avec la nouvelle condition « BTKE Van Rijn prolongé » se sont montrés satisfaisants aussi bien sur le taux d'abattement des particules (efficacité) que pour les zones préférentielles de dépôts. En effet, le modèle permet de reproduire l'efficacité avec une erreur absolue de l'ordre de $5 \%$ dans le cas de dépôts sur l'ensemble du fond. Dans le cas de dépôts localisés, les zones préférentielles sont reproduites avec une bonne fidélité et l'efficacité est évaluée avec une erreur absolue de l'ordre de $10 \%$ (hors cas de particules très peu denses).

$\mathrm{Au}$ final, un outil numérique est disponible et validé pour évaluer les performances d'un ouvrage de décantation vis-à-vis de l'abattement des matières en suspension. L'application première de cet outil intervient dans le cadre du projet SIMPLUV, dont une des ambitions est le développement d'un ouvrage de décantation préfabriqué. L'outil est utilisé pour tester différentes formes et aménagements de l'ouvrage dans le but d'y maximiser les dépôts. Une phase d'expérimentation sur un modèle physique du décanteur à l'échelle 1 viendra conforter les développements réalisés par l'outil numérique et permettra la validation du modèle sur un ouvrage réel (sans similitude). Le domaine d'application de l'outil se veut néanmoins plus large que le projet SIMPLUV. Celui-ci peut par exemple être utilisé en phase de conception ou de réhabilitation d'un bassin de plus grande taille (de l'ordre du millier de mètres cube) pour proposer des formes et des aménagements permettant de maximiser l'abattement des matières en suspension ou de réduire le volume pour un niveau de performance donné. 
Plus généralement, les perspectives scientifiques de ce travail sont les suivantes. Du point de vue numérique, il serait intéressant de procéder à une étude plus poussée de la répartition spatiale des dépôts. La comparaison qualitative (visuelle) pourrait être complétée par analyse quantitative à travers, par exemple, le pourcentage de masse surfacique ou un autre paramètre représentatif de l'épaisseur de dépôt. Du point de vue expérimental, cela nécessiterait de réaliser des mesures permettant la création d'une base de données expérimentales sur les hauteurs de sédiments sur le fond. Des mesures de ce type, basées sur une méthode ultrasonore, ont été initiées lors de la campagne expérimentale du projet SIMPLUV [Isenmann 2016].

\section{RÉFÉRENCES}

Adamsson Å, Stovin V, Bergdahl L (2003) - Bed shear stress boundary condition for storage tank sedimentation. Journal of Environmental Engineering, 129(7), 651-658.

Adamsson Å, Bergdahl L, Lyngfelt S (2005) - Measurement and three-dimensional simulation of flow in a rectangular detention tank. Urban Water Journal, 2(4), 277-287.

Amsden Aa, O'rourke PJ, Butler Td (1989) - KIVA-II: a computer program for chemically reactive flows with sprays. Loas Alamos National Laboratory, LA-AA560-MS

Ashley Rm, Bertrand-Krajewski Jl, Hvitved-Jacobsen T, VERBANCK M (2004) - Solids in sewers: characteristics, effects and control of sewer solids and associated pollutants. IWA Publishing.

BRESSY A (2010) - Flux de micropolluants dans les eaux de ruissellement urbaines : effets de différents modes de gestion à l'amont. Thèse de doctorat, Université Paris-Est, Paris, France.

BROWNLIE WR (1981) - Prediction of flow depth and sediment discharge in open channels. Report No. KH-R-43A, W. M. Keck Laboratory of Hydraulics and Water Resources, California Institute of Technology, Pasadena, California, USA.

Buffington J (1999) - The legend of A. F. Shields. Journal of Hydraulic Engineering, 125(4), 376-387.

Deshpande Ss, Anumolu L, Trujillo Mf (2012) - Evaluating the performance of the two-phase flow solver interFoam. Computational Science \& Discovery, 5, 014016.

Dufresne M, Terfous A, Ghenaim A, Poulet J, Vazquez J (2007) - Modélisation 3D du transport et du dépôt de particules dans un pilote de bassin d'orage. La Houille Blanche, 5, 95-100.

DUFRESNE M (2008) - La modélisation $3 D$ du transport solide dans les bassins en assainissement : du pilote expérimental à l'ouvrage réel. Thèse de doctorat, Université Louis Pasteur, Strasbourg, France.

Dufresne M, Terfous A, Ghenaim A, Poulet J, Vazquez J (2008) - Prévoir l'efficacité des bassins d'orage par la modélisation 3D : du bassin expérimental à l'ouvrage réel. La Houille Blanche, 5, 92-98.

Dufresne M, Vazquez J, Terfous A, Ghenaim A, Poulet J (2009) - Experimental investigation and CFD modelling of flow, sedimentation, and solids separation in a combined sewer detention tank. Computer \& Fluids, 38(5), 1042-1049.

Dufresne M, Dewals BJ, Erpicum S, Archambeau P, Pirotton M (2010) - Classification of flow patterns in rectangular shallow reservoir. Journal of Hydraulic Research, 48(2), 197-204.
Frey P, Champagne Jy, Morel R, Gay B (1993) - Hydrodynamics fields and solid particles transport in a settling tank. Journal of Hydraulic Research, 31(6), 736-776.

Graf Wh, Altinakar Ms (2000) - Hydraulique fluviale, Traité de Génie Civil. Presses Polytechniques et Universitaires Romandes, 16.

HiRT Cw, Nichols BD (1981) - Volume of Fluid (VOF) Method for the Dynamics of Free Boundaries. Journal of Computational Physics, 39, 201-225.

IsENMANN G. (2016) - Approche Euler-Lagrange pour la modélisation du transport solide dans les ouvrages de décantation. Thèse de doctorat, Université de Strasbourg, France.

JarRell-Smith S, Friedrich CT (2011) - Size and settling velocities of cohesive flocs and suspended sediments aggregates in a trailing suction hopper dredge plume. Continental Shelf Research, 31(2011), 550-563.

Kantoush SA (2008) - Experimental study on the influence of the geometry of shallow reservoirs on flow patterns and sedimentation by suspended sediments. Thèse de doctorat. Ecole Polytechnique Fédérale de Lausanne, Suisse.

MaXey Mr, Riley JJ (1983) - Equation of motion for a small rigid sphere in a nonuniform flow. Physics of Fluids, 26(4), 883-889.

Morsi Sa, Alexander Aj (1972) - An investigation of particle trajectories in two-phase flow systems. Journal of Fluid Mechanics, 55, 196-208.

Openfoam (2016) - Dépôts officiels du logiciel. [en ligne]. Disponible sur : https://github.com/OpenFOAM [consulté le 1 juin 2016].

Pope Nd, Widdows J, BRinsLey Md (2006) - Estimation of bed shear stress using the turbulent kinetic energy approach - A comparison of annular and field data. Cont. Shelf Res, 26, 959-970.

RUSCHE H (2002) - Computational fluid dynamics of dispersed two-phase flows at high phase fraction. Thèse de doctorat. Imperial College of Science, Technology and Medecine, Londres, Royaume-Uni.

Stovin VR, SAUl AJ (1994) - Sedimentation in storage tank structures. Water Science and Technology, 29(1-2), 363-372.

Stovin VR, SAUL AJ (1996) - Efficiency prediction for storage chambers using computational fluid dynamics. Water Science and Technology, 33(9), 163-170.

STOVIN VR (1996) - The prediction of sediment deposition in storage chambers based on laboratory observations and numerical simulation. Thèse de doctorat, Université de Sheffield, Royaume-Uni.

TORRES A (2008) - Décantation des eaux pluviales dans un ouvrage réel de grande taille : éléments de réflexion pour le suivi et la modélisation. Thèse de doctorat. INSA de Lyon, France.

VAN RIJN L (1984) - Sediment transport, part II: suspended load transport. Journal of Hydraulic Engineering, 110(11), 1613-1638.

Versteeg HK, Malalasekera W (2007) - An introduction to computational fluid dynamics. Prentice Hall.

Yan H, Lipeme Kouyi G, Gonzalez-Merchan C, Becouze-Lareure C, Sebastian C, Barraud S, Bertrand-Krajewski Jl (2014) - Computational fluid dynamics modelling of flow and particulate contaminants sedimentation in an urban stormwater detention and settling basin. Environmental Science and Pollution Research, 21(8), 5347-5356. 